\title{
Ice growth in a spherical cavity of a porous medium
}

\author{
Ioanna VLAHOU, M. Grae WORSTER \\ Institute of Theoretical Geophysics, Department of Applied Mathematics and Theoretical Physics, \\ Centre for Mathematical Sciences, University of Cambridge, Wilberforce Road, Cambridge CB3 OWA, UK \\ E-mail: I.Vlahou@damtp.cam.ac.uk
}

\begin{abstract}
We consider an idealized problem of a sphere of ice growing symmetrically in a spherical cavity within a porous rock in order to identify and quantify different physical mechanisms that can result in fracturing the rock. We show that if the permeability of the rock is very small then high pressures can develop in the cavity as the water inside it expands on freezing. However, given typical permeabilities of most rocks, the pressure is relieved by flow out of the cavity through the rock pores. When ice fills the cavity, there remains a microscopic film of water separating the ice from the rock, owing to disjoining forces, and these forces can stress the rock and have the potential to fracture it. The elastic pressure in the rock depresses the freezing temperature, which can limit the potential for fracturing. This simple example reveals the important interactions between disjoining forces, elasticity and fluid flow in determining the pressure exerted during freezing of water-saturated cavities in rocks.
\end{abstract}

\section{INTRODUCTION}

Fracturing occurs when water in saturated rocks freezes. It is an important problem for both engineers and scientists, as it can affect building and highway construction, water supplies and gas and oil pipelines. Its importance in the development of landscapes is also widely recognized (Washburn, 1980). It is often assumed that the volumetric expansion $(\sim 9 \%)$ of freezing water is the fundamental mechanism underlying the fracturing of rock by ice. However, if expansion played an important role in rock fracturing, there would be a critical saturation level ( $\sim 91 \%)$, below which fracturing would not occur. This is not supported by experimental data (Walder and Hallet, 1985).

It is reasonable to expect that frost weathering is governed by mechanisms similar similar to those for frost heave in soils. The latter has been studied extensively and it is known that it is water migration from unfrozen regions towards the solidification fronts that causes ice lenses to grow (Taber 1929, 1930). Here, like Walder and Hallet (1985), we assume that ice-filled cavities in rocks play the role of ice lenses in soils. Disjoining (intermolecular) forces between the ice and the rock lower the pressure in unfrozen water films adjacent to the ice surface, which draws water in from the surrounding saturated medium. These same forces cause the ice-filled cracks to widen. Thus, the same ideas that have been used to study frost heave in soils can be applied to the freezing of water-saturated rocks. The difference lies in the way each medium deforms under the forces exerted by the ice; soil particles can be rejected from the solidification front if the freezing is slow enough, while the pressure exerted on the rock deforms it elastically.

The rate of fracturing, or frost heave, depends on the ability of water to flow to the freezing front, and hence on the size of the pores. There are two factors that act in opposing ways: the magnitude of the forces and the water mobility. Finer soils generate bigger forces, but water transport is limited owing to low permeability, and hence the heave is slower. It is the balance of these processes that maximizes heaving. Frost-susceptible soils are usually silts which have grain sizes of $\sim 10 \mu \mathrm{m}$, between sand $(\sim 100 \mu \mathrm{m})$ and clay (below $\sim 1 \mu \mathrm{m}$ ). Similarly, limestones and sandstones (with permeabilities of around $10^{-10}-10^{-13} \mathrm{~cm}^{2}$; see
Bear, 1988) are more prone to fracturing than more permeable rocks. In the case of very impermeable rocks, such as granite, we see below that the expansion of the freezing water can play a role.

Walder and Hallet (1985) developed a model for the fracture of the rock during freezing. They recognized the importance of the flow of water towards the solidification front as well as the existence of thin films separating the ice and the rock. They discussed how these films exert an 'attractive force' on the pore-water (hence the flow towards the ice front) and a disjoining pressure that pushes the ice and the rock apart. This disjoining pressure was then equated to the ice pressure, under the assumption that the water pressure is uniform and negligible compared to the ice pressure. They showed that the fastest growth rate occurs at temperatures in the range -4 to $-15^{\circ} \mathrm{C}$. In colder systems, the transport of water is very difficult, owing to ice forming a frozen fringe in the pores surrounding a crack. For temperatures closer to $0^{\circ} \mathrm{C}$, there is not enough pore ice to raise the pressure sufficiently to create crack growth in the pores surrounding the fracture. The same fracture model was used by Murton and others (2006) for their numerical simulations. The idea of disjoining pressure is used in this paper, incorporated into a rigorous mathematical model. This model allows for variations of the water pressure, which plays a very important role in the water flow towards the solidification front. We do not consider a frozen fringe but instead focus attention on the effect that the permeability of the rock has on the water mobility and the pressure rise in the cavity.

In this paper, we are interested in the effects that expansion and disjoining forces have on the elastic pressure which eventually causes fracturing. We find that the effect of expansion is negligible in most cases. Therefore, when we want to restrict attention to the effect of disjoining forces we ignore expansion by setting the density of the ice, $\rho_{\mathrm{s}}$, equal to the density of the water, $\rho_{\mid}$. We concentrate on the physical mechanisms associated with the disjoining pressure, using a model based on van der Waals forces, and showing that it has to balance the pressure difference between the ice and the water. The results of this study give us a useful insight into the mechanisms involved in the 


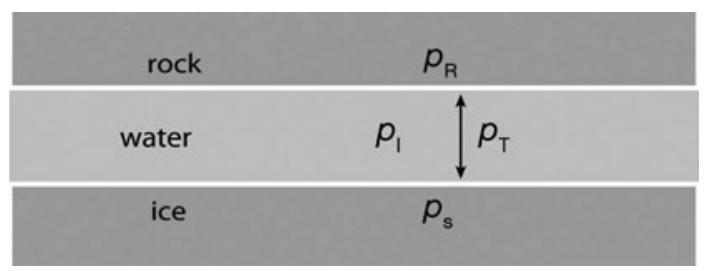

Fig. 1. Water fills the gap between ice and rock. The disjoining pressure, $p_{\mathrm{T}}$, plus the water pressure, $p_{\mathrm{l}}$, balance the solid pressure, $p_{\mathrm{s}}=p_{\mathrm{R}}$.

fracturing of rocks and how they contribute to the pressure fields within rocks and ice-filled cavities within them.

\section{PRE-MELTING DYNAMICS}

Classical thermodynamics can be used to describe the process of solidification, i.e. the change from liquid to solid state. At a phase boundary in equilibrium, the specific Gibbs free energy is the same in the two phases either side. We consider the independent thermodynamic variables temperature, $T$, and pressure, $p$. By considering small departures from a reference state $\left(T_{\mathrm{m}}, p_{\mathrm{m}}\right)$ to a state that has $\left(T, p_{\mathrm{s}}\right)$ in the solid state and $\left(T, p_{1}\right)$ in the liquid state (since the temperature is continuous across the boundary) it can be shown that

$$
\frac{\rho_{\mathrm{s}} L \Delta T}{T_{\mathrm{m}}}=\left(p_{\mathrm{s}}-p_{\mathrm{l}}\right)+\left(p_{\mathrm{l}}-p_{\mathrm{m}}\right)\left(1-\frac{\rho_{\mathrm{s}}}{\rho_{\mathrm{l}}}\right),
$$

where $\Delta T=T_{\mathrm{m}}-T$ (Wettlaufer and Worster, 2006). Equation (1) is known as the Gibbs-Duhem equation. The densities of the solid and liquid states are $\rho_{\mathrm{s}}$ and $\rho_{\mathrm{l}}$, respectively, while $L$ is the latent heat of fusion per unit mass released during solidification.

If there is no pressure difference across the solid-liquid interface, so $p_{\mathrm{s}}=p_{\mathrm{l}}$, we obtain the Clausius-Clapeyron relation

$$
p_{\mathrm{l}}-p_{\mathrm{m}}=\left(\frac{1}{\rho_{\mathrm{s}}}-\frac{1}{\rho_{\mathrm{l}}}\right)^{-1} \frac{L \Delta T}{T_{\mathrm{m}}}
$$

The effect that a pressure applied on a freezing surface has on the freezing temperature depends on the densities of the liquid and the solid state. Water is a special case, since it is denser than ice. Therefore, a rise in pressure on ice causes it to melt. This well-known effect, pressure melting, is significant in the scenarios we consider below.

A pressure difference across the interface, described by the first term on the right-hand side of Equation (1), can arise due to intermolecular forces acting between the solid and another material. The existence of melt on the surface of a solid below its freezing temperature as a result of intermolecular interactions across the boundaries is called pre-melting. It occurs either at the vapour interface (surface melting), against a foreign substrate (interfacial melting) or at the interface between two crystallites of the same substance (grain-boundary melting) (Wettlaufer and Worster, 2006).

Interfacial melting can be induced by a variety of intermolecular forces. In the case of van der Waals interactions, for example, when a solid and a foreign substrate are separated by a thin film of melt of thickness $h$ (Fig. 1), the intermolecular forces give rise to a pressure
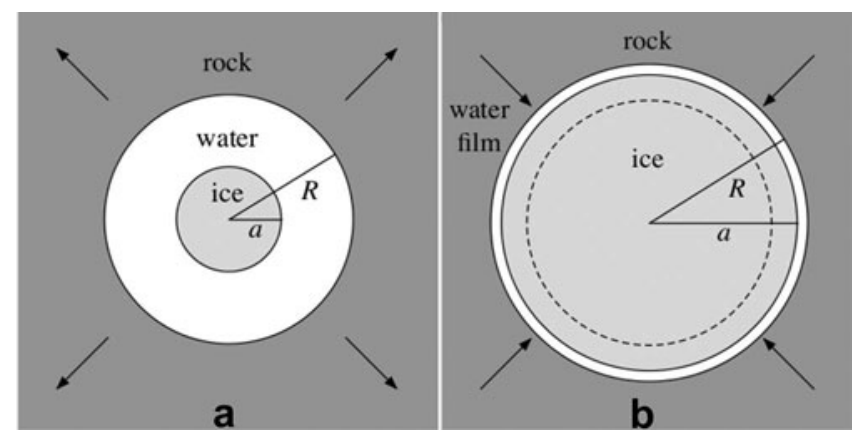

Fig. 2. (a) Ice growing inside a water-saturated spherical cavity. The expansion of the water as it freezes drives unfrozen water out of the cavity through the porous medium. (b) The later stage, where disjoining forces push the rock and the ice apart. Water flow is reversed because the liquid pressure, $p_{\mathrm{I}}=p_{\mathrm{s}}-p_{\mathrm{T}}$, is negative.

between the solid and the substrate of the form

$$
p_{\text {T }}=\frac{A}{6 \pi h^{3}},
$$

where $A$ is the effective Hamaker constant (Löwen and others, 1989), and depends on the dielectric properties of all three materials involved and can have either sign. A negative sign leads to an attraction force and rupturing of any intervening liquid film. We are interested in the cases where $A$ is positive and hence the pressure between the solid and the substrate is disjoining. The balance of pressures across the liquid film gives

$$
p_{\mathrm{l}}=p_{\mathrm{s}}-p_{\mathrm{T}}
$$

which describes the dependence of the liquid pressure on the thickness of the pre-melted film (through $p_{T}$ ) and on the elastic forces between the ice and the rock. As we describe below, the net force between ice and rock depends only on the local temperature and is independent of the type of intermolecular interactions, which only determines the film thickness.

\section{SPHERICAL MODEL}

As a means to illustrate and understand the different physical mechanisms involved when ice forms inside a cavity of a porous elastic rock, we consider the geometrically simple case of a spherical cavity, illustrated in Figure 2. This unrealistic idealization captures the essential interactions and the magnitudes of the pressures involved. We consider a system supercooled to some uniform temperature $T_{\infty}<T_{\mathrm{m}}$, where $T_{\mathrm{m}}$ is the melting temperature of the ice measured at pressure $p_{\mathrm{m}}=p_{\infty}$. We are interested in finding out how the radius of the solid ice and the pressure in the cavity evolve with time and also how the different parameters of the problem affect the solidification process.

We will see that there are two main stages of growth. The first is the expansion regime, shown in Figure 2a, where the ice fills the cavity and water flows away from the solidification front as it expands on freezing. The expansion causes pressure to build up inside the cavity which can be partially relieved by water flowing through the porous medium. Therefore, the magnitude of this pressure depends on the permeability of the rock. For a very permeable rock the water will flow easily and the pressure build-up will be negligible. The second regime is when the ice has almost 
filled the cavity and is characterized by strong intermolecular forces between the ice and the rock, acting through a thin water film separating the two. The ice formation has now slowed considerably and the main pressure contribution comes from the disjoining forces. These forces cause the cavity to expand, widening the gap between the ice and the rock and hence causing water to flow towards the ice front, as shown in Figure $2 \mathrm{~b}$. We show below that this is the important regime, since the pressures appearing here are, for most rocks, much larger than the pressure build-up through expansion and, furthermore, they cannot be relieved.

\section{Governing equations}

We assume that solidification begins at the centre of the supercooled cavity and that the solid ice grows in a spherical shape with radius $a(t)$. As the water freezes, it expands, causing flow away from the solidification front as shown in Figure $2 \mathrm{a}$. The water tries to escape the cavity through the porous medium in which the flow is controlled by its permeability. The resistance to flow through the porous medium causes the pressure in the cavity to rise. The increased pressure on the freezing interface depresses the freezing temperature according to the Clausius-Clapeyron equation (2), and hence the flow through the porous medium controls the rate of solidification. We have ignored the curvature of the solidifying front, which would create a pressure difference across it and hence a further depression of the melting temperature. It can be shown that the effect of curvature, known as the Gibbs-Thompson effect, is negligible for cavities with radii greater than $\sim 10^{-5} \mathrm{~cm}$. We assume the flow in the cavity is slow and that the temperature field is quasi-steady so

$$
\nabla^{2} T=0
$$

We also assume, for simplicity, that the thermal properties of ice, water and rock are identical. The temperature is bounded at the origin (the centre of the cavity) and $T \rightarrow T_{\infty}$ as $r \rightarrow \infty$ (i.e. in the rock far from the cavity). The spherically symmetric solution to this is

$$
T_{\mathrm{s}}=T^{\mathrm{I}}(t) \text { and } T_{\mathrm{l}}=T_{\infty}+\frac{T^{\mathrm{I}}(t)-T_{\infty}}{r} \mathrm{a}(t),
$$

where $T_{\mathrm{s}}$ and $T_{1}$ are the temperature fields in the solid ice and the water, respectively, $T^{1}$ is the temperature at the solidifying interface given by the Clausius-Clapeyron equation (2) and $a(t)$ is the radius of the ice. When water solidifies, there is a release of latent heat of fusion, which has to be transported away in order for the process to continue. This balance of heat is described by the Stefan condition

$$
\rho_{\mathrm{s}} L \dot{a}(t)=\mathbf{n} \cdot \mathbf{q}_{\mathrm{l}}-\mathbf{n} \cdot \mathbf{q}_{\mathrm{s}},
$$

where $\mathbf{q}_{\mathrm{s}}=-k_{\mathrm{s}} \nabla T$ and $\mathbf{q}_{\mathrm{l}}=-k_{\mathrm{l}} \nabla T$, evaluated at $r=a(t)$, and $k_{\mathrm{s}}$ and $k_{\mathrm{l}}$ are the thermal conductivities of ice and water, respectively. The Stefan condition expresses the fact that the rate of release of latent heat per unit area is equal to the net heat flux away from the interface. Combining Equations (6) and (7) we obtain

$$
\mathbf{n} \cdot \mathbf{q}_{\mathrm{I}_{\mathrm{r}=\mathrm{a}}}=\frac{\mathbf{T}^{\mathbf{l}}-\mathbf{T}_{\infty}}{\mathbf{a}}
$$

governing the rate of solidification.

The interfacial temperature, $T^{1}$, is determined by the pressure in the cavity, which is controlled by the flow in the surrounding porous medium. Such flow is described by Darcy's equation,

$$
\mu \mathbf{u}=-\Pi \nabla p_{l}
$$

where $\Pi$ is the permeability of the rock, $\mu$ is the dynamic viscosity and the incompressibility constraint is

$$
\nabla \cdot \mathbf{u}=0 .
$$

Note that we ignore any motion of the rock in the relative flow equation. Without loss of generality, we consider the pressures to be relative to the far-field pressure in the rock, $p_{\infty}$. Therefore, the pressure $p \rightarrow 0$ as $r \rightarrow \infty$ and is continuous across the cavity boundary $r=R(t)$, where the cavity has radius $R(t)$. We expect the flow in the cavity to be slow enough that the pressure gradient there is negligible compared to that in the porous medium and hence we assume the cavity pressure to be constant. Mass conservation, expressed as

$$
\begin{aligned}
& \text { (rate of change of mass in sphere) } \\
& =-(\text { mass flux out of sphere), }
\end{aligned}
$$

shows the velocity to be

$$
u(r, t)= \begin{cases}\frac{\Delta \rho}{\rho_{\mathrm{l}}} \frac{a^{2} \dot{a}}{r^{2}} & r<R, \\ \frac{\Delta \rho}{\rho_{\mathrm{l}}} \frac{a^{2} \dot{a}}{r^{2}}-(1-\phi) \frac{R^{2} \dot{R}}{r^{2}} & r>R,\end{cases}
$$

where $\Delta \rho=\rho_{\mathrm{l}}-\rho_{\mathrm{s}}$ and $\phi$ is the porosity of the rock. In the pores, the water pressure is related to the flow velocity by Darcy's equation (9). The water pressure inside the cavity can be found from the value at the cavity boundary. Therefore

$$
p_{l}(r, t)= \begin{cases}\frac{\mu}{\Pi}\left[\frac{\Delta \rho}{\rho_{\mathrm{l}}} \frac{a^{2} \dot{a}}{R}-(1-\phi) R \dot{R}\right] & r<R \\ \frac{\mu}{\Pi}\left[\frac{\Delta \rho}{\rho_{\mathrm{l}}} \frac{a^{2} \dot{a}}{r}-(1-\phi) \frac{R^{2} \dot{R}}{r}\right] & r>R .\end{cases}
$$

The second term in each expression, involving $\dot{R}$, represents the opening of the cavity under pressure. Water flows freely through the region previously occupied by the porous medium, hence the water pressure is relaxed.

\section{Expansion}

The first stage of the process is the expansion stage, in which the solid ice is still small compared to the cavity so there is no interaction between ice and rock. For simplicity, we initially take $R(t)=R_{0}$, i.e. constant. The effect of an elastic cavity is considered in the next subsection, where we see that this is an accurate approximation for the early stages of expansion, at least for most types of rocks. The second term in the expression for pressure, Equation (13), vanishes, and hence the pressure in the porous medium is simply given by

$$
p_{\mathrm{l}}=\frac{\mu \Delta \rho}{\Pi \rho_{\mathrm{l}}} \frac{a^{2} \dot{a}}{r} \quad \text { for } \quad r>R,
$$

and the uniform pressure in the cavity is

$$
p_{l}=\frac{\mu \Delta \rho}{\Pi \rho_{l}} \frac{a^{2} \dot{a}}{R} \text { for } r<R .
$$

Equation (15) can be combined with the Clausius-Clapeyron relation (2) to give the interface temperature in terms of the 
Table 1. Some typical values for the parameters used in the analysis

\section{Parameter}

Value

Latent heat, $L$

Density of ice, $\rho_{\mathrm{s}}$

Density of water, $\rho_{\mathrm{l}}$

Thermal conductivity, $k_{\text {I }}$

Dynamic viscosity, $\mu$

Melting temperature, $T_{\mathrm{m}}$

Elastic modulus, $E$

$$
\begin{gathered}
334 \times 10^{3} \mathrm{~m}^{2} \mathrm{~s}^{-2} \\
0.92 \times 10^{3} \mathrm{~kg} \mathrm{~m}^{-3} \\
10^{3} \mathrm{~kg} \mathrm{~m}^{-3} \\
2 \mathrm{~kg} \mathrm{~m} \mathrm{~s}^{-3} \mathrm{~K}^{-1} \\
1.79 \times 10^{-3} \mathrm{~kg} \mathrm{~m}^{-1} \mathrm{~s}^{-1} \\
273 \mathrm{~K} \\
5 \times 10^{10} \mathrm{~kg} \mathrm{~m}^{-1} \mathrm{~s}^{-2}
\end{gathered}
$$

ice radius as

$$
T^{1}=T_{\mathrm{m}}\left[1-\frac{\mu(\Delta \rho)^{2}}{\rho_{\mathrm{s}} \rho_{\mathrm{l}}^{2} \Pi L} \frac{\mathrm{a}^{2} \dot{a}}{R}\right] .
$$

Putting this together with the heat-balance equation (8), we find a differential equation for the ice radius, $a(t)$,

$$
a^{2} \dot{a}+K R a \dot{a}=\frac{k_{l} \Delta T}{\rho_{\mathrm{s}} L} K R,
$$

where $\Delta T=T_{\mathrm{m}}-T^{\mathrm{l}}$ and

$$
K=\frac{\rho_{\mathrm{s}}^{2} \rho_{l}^{2} L^{2} \Pi}{k_{\mathrm{l}} \mu(\Delta \rho)^{2} T_{\mathrm{m}}}
$$

is a dimensionless parameter proportional to the permeability. The first term on the left-hand side of Equation (17) represents the pressure-melting effect, while the second term comes from the heat balance and represents the flow of latent heat away from the solidification front. They both affect the rate of solidification: high pressure on the ice causes depression of the freezing temperature and hence causes the process to slow down, while latent heat transport is necessary for the solidification to continue.

The two terms on the left-hand side of Equation (17) are comparable when $a \sim K R$, where the parameter $K \sim 10^{15} \times \Pi \mathrm{cm}^{-2}$. Typical permeability values vary from $10^{-3} \mathrm{~cm}^{2}$ for very permeable media, such as highly fractured rocks, to $10^{-12}-10^{-15} \mathrm{~cm}^{2}$ for rocks like unfractured limestone or granite (Bear, 1988). If $K \gg 1$, the two terms are never comparable, since the radius of the ice, $a$, is smaller than the radius of the cavity, $R$, and hence the porous medium does not affect the ice growth significantly. The ice then grows proportional to $t^{1 / 2}$. For highly impermeable rocks like limestone or granite, both terms in Equation (17) contribute, especially at the later stages of solidification. A low permeability results in the flow through the porous medium being highly restricted. Hence, the pressure inside the cavity rises enough to have a considerable effect on the melting temperature and therefore on the ice growth. If we allow for the cavity to behave elastically, high cavity pressures will deform it and hence our assumption of constant cavity radius fails. In return, such a deformation will relax the water pressure in the cavity. The balance of these two processes and the magnitude of their effects on the water pressure will determine the growth rate, as we see in the next subsection.

Is the pressure high enough to fracture a rock? The maximum pressure in the cavity occurs when $a(t)=R$, i.e.

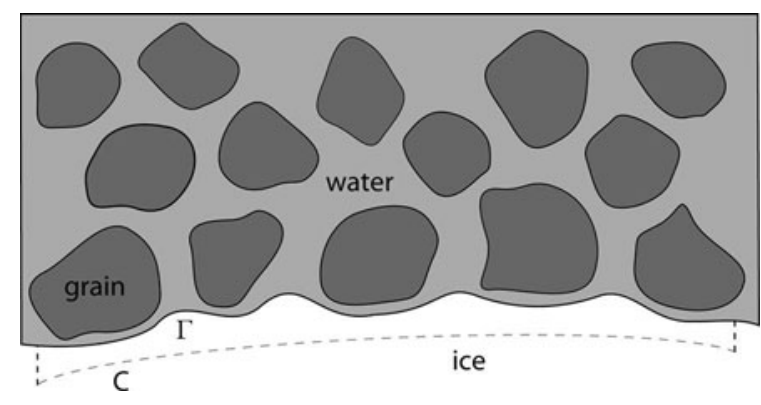

Fig. 3. A cross-section near the cavity/porous-medium boundary. The ice expands into the free space of the porous medium, while it is separated from the grains by a thin film of pre-melted water. The macroscopic, smooth surface, $C$, can replace the corrugated ice/ water interface.

when the ice has filled the cavity, and is equal to

$$
p_{\max }=\frac{\rho_{\mathrm{s}} \rho_{\mathrm{l}} L \Delta T}{\Delta \rho T_{\mathrm{m}}} \frac{R}{1+K} \approx \frac{120 \mathrm{~atm}}{1+1.4 \times \Pi \times 10^{15} \mathrm{~cm}^{-2}},
$$

for $T_{\mathrm{m}}=0^{\circ} \mathrm{C}, \Delta T=1^{\circ} \mathrm{C}$ and $p_{\mathrm{m}}=1 \mathrm{~atm}$ (see Table 1 ). Hence, the pressure in the cavity varies from $10^{-3} \mathrm{~atm}$, which is negligible, for a rock like sandstone with permeabilities $\sim 10^{-10} \mathrm{~cm}^{2}$, to $10^{2} \mathrm{~atm}$, which is more than sufficient to fracture a rock, for permeabilities of $10^{-15} \mathrm{~cm}^{2}$ (e.g. granite). These have been calculated for a cavity of radius $R=1 \mathrm{~cm}$. It is clear from Equation (19) that inside smaller cavities these would be smaller pressures. Therefore, to have an important pressure build-up during the expansion regime, we need a cavity of radius of at least $1 \mathrm{~cm}$ in a rock of very low permeability $\left(\Pi=10^{-14}-10^{-15} \mathrm{~cm}^{2}\right)$ like granite.

\section{Pre-melting}

When the ice has almost filled the cavity, the distance from the grains is small enough for thermomolecular repulsion forces between the two solids to be important. These forces give rise to a disjoining pressure, $p_{\mathrm{T}}$, which has to balance the pressure difference between the water and both of the surrounding solids. Close to the cavity boundary the ice can protrude inside the pores, making the ice front highly curved, as shown in Figure 3. Accounting for curvature, we find

$$
p_{\mathrm{s}}-p_{\mathrm{l}}=p_{\mathrm{T}}+\kappa \gamma,
$$

where $\kappa$ is the curvature of the ice boundary and $\gamma$ is the surface tension. The disjoining pressure depends on the thickness, $h$, of the water film between the ice and the rock. For example, for non-retarded van der Waals forces it is given by $p_{\mathrm{T}}=A / 6 \pi h^{3}$, where $A$ is the Hamaker constant.

The net thermomolecular force on the rock, arising from intermolecular interactions, can be computed as

$$
\mathbf{F}_{\mathrm{T}}=-\int_{\Gamma} p_{\mathrm{T}} \mathrm{d} \Gamma=-\int_{\Gamma}\left(\rho_{\mathrm{s}} L \frac{T_{\mathrm{m}}-T^{\mathrm{l}}}{T_{\mathrm{m}}}-\gamma \kappa\right) \mathrm{d} \Gamma,
$$

where the surface $\Gamma$ is, as shown in Figure 3, the surface of the ice.

We can close $\Gamma$ by adding the dashed surface of area $C$ which we assume is at $r=R$, following Rempel and others (2004). The integral of the curvature, $\kappa$, over a closed surface vanishes and we can assume that the surface $C$ is approximately flat locally. Then, implementing the divergence 

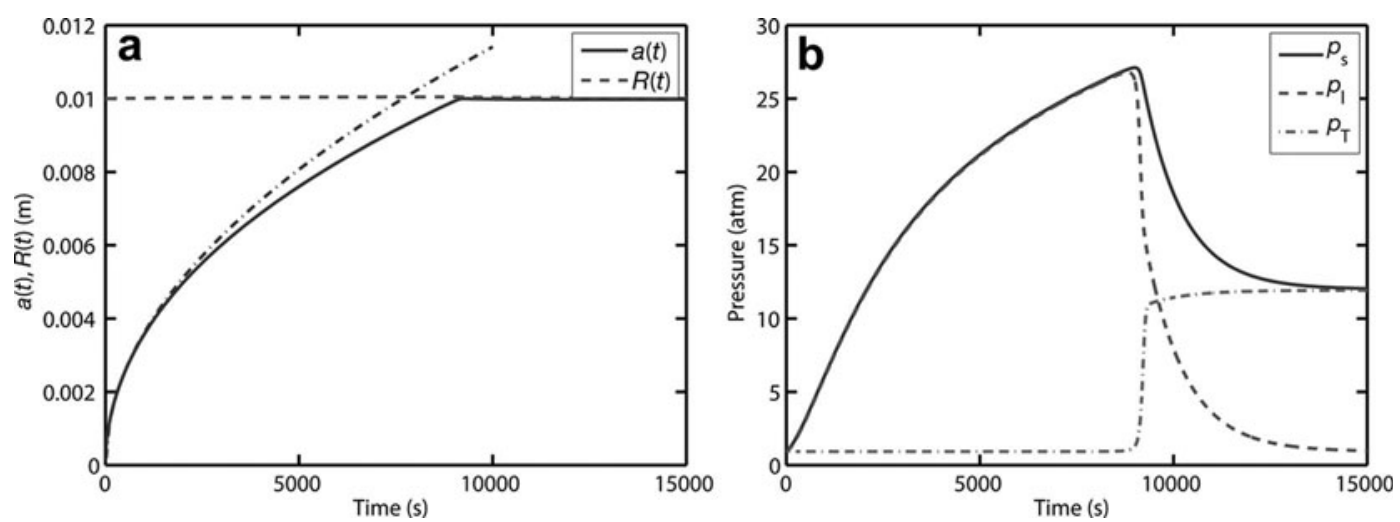

Fig. 4. Results for the evolution of (a) radii and (b) pressure values in granite. (a) The radius of the cavity is represented by the dashed curve, the radius of the ice by the solid curve and the dot-dashed curve shows the approximate result from the expansion regime. (b) The solid curve is the solid pressure, the dashed curve is the water pressure and the dot-dashed curve the disjoining pressure.

theorem, we obtain

$$
\mathbf{F}_{\mathrm{T}}=-\frac{\rho_{\mathrm{s}} L}{T_{\mathrm{m}}} \int_{V} \nabla\left(T_{\mathrm{m}}-T^{\mathrm{I}}\right) \mathrm{d} V+\frac{\rho_{\mathrm{s}} L}{T_{\mathrm{m}}} \int_{C}\left(T_{\mathrm{m}}-T^{\mathrm{I}}\right) \mathrm{d} \mathbf{C} .
$$

Since the temperature in the ice is uniform, the first term on the right-hand side vanishes, while the second term simply gives

$$
\mathbf{F}_{\mathrm{T}}=\rho_{\mathrm{s}} L C \frac{T_{\mathrm{m}}-T^{1}}{T_{\mathrm{m}}} \mathbf{n},
$$

where $\mathbf{n}$ is the normal to the surface $C$. It is important to note here that the net disjoining force is independent of the curvature and independent of the type and strength of interactions that give rise to the disjoining pressure, $p_{\text {T }}$. Hence our choice of van der Waals interactions as a model for the intermolecular forces does not affect the results. Moreover, it only depends on the approximated boundary, $C$, and not on the microscopically complicated surface $\Gamma$. Hence, we are justified in treating the ice/water/rock boundary by averaging over the pore scale.

We assume the porous medium is elastic and use Hooke's law to represent the deformation of it under pressure

$$
p_{\mathrm{s}}(R, t)=E\left(\frac{R_{0}}{R}-1\right)
$$

where $R_{0}$ is the initial radius of the cavity and $E$ is the modulus of elasticity of the rock. The cavity starts expanding under the pressure that the growing ice exerts on its boundary, which, in turn, relaxes the pressure. The expansion of the cavity causes more water to be drawn in towards the solidification front. The water freezes and the process continues until, ultimately, the system reaches an equilibrium where the disjoining pressure is balanced by the restoring force exerted by the deformed rock. The equation for the evolution of the cavity radius comes from the GibbsDuhem relation combined with the heat balance equation (8) to give

$$
\begin{aligned}
a^{2} \dot{a}+K R a \dot{a}= & \frac{k_{\mathrm{l}} \Delta T}{\rho_{\mathrm{s}} L} K R+\frac{\rho_{\mathrm{l}}}{\Delta \rho}(1-\phi) R^{2} \dot{R} \\
& -\frac{\rho_{\mathrm{l}}^{2}}{(\Delta \rho)^{2}} \frac{\Pi}{\mu} \frac{A R}{6 \pi(R-a)^{3}},
\end{aligned}
$$

which is similar to Equation (17) with two extra terms, representing the cavity expansion and the disjoining pressure. The latter balances the pressure difference across the interface, $p_{\mathrm{s}}-p_{\mathrm{l}}$, to give a second equation for the system,

$$
\frac{A}{6 \pi(R-a)^{3}}=E\left(\frac{R}{R_{0}}-1\right)-\frac{\mu \Delta \rho}{\rho_{1} \Pi R} a^{2} \dot{a}+\frac{\mu(1-\phi)}{\Pi} R \dot{R} .
$$

\section{Results}

The system of differential equations (25) and (26) was solved using the Matlab ${ }^{\text {TM }}$ solver ode15s. Figures $4-6$ show results for the evolution of the radii and pressures with time. In all of these results, the effect of disjoining forces has been exaggerated by using a larger value for the Hamaker constant, $A$. The actual value of the constant is $10^{-18} \mathrm{~J}$ while we have used values of $O(1)$ in order to make the gap between the ice and the rock visible. The qualitative results are the same, and the quantitative consequences are discussed in more detail below. We start by showing the results for a very impermeable rock, like granite (Fig. 4), with permeability $\Pi \sim 10^{-15} \mathrm{~cm}^{2}$. There are three distinct stages. The first corresponds to the expansion regime discussed above. In the analysis there, we assumed that the cavity does not deform and hence we found ice growth with rate proportional to $t^{1 / 2}$ (Fig. 4; dot-dashed curve). We notice that the cavity expands most in this regime and consequently the two predictions diverge at larger ice radii. This is explained by considering that, for such impermeable rocks, the flow of water away from the solidification front is restrained, which results in a high water pressure inside the cavity, and hence deformation of the cavity. The high pressure on the solidification front subsequently results in depression of the freezing temperature and hence slower growth, as shown in Figure 4. The second regime represents a quick transition from the expansion stage to the premelting stage, with both the solid and the liquid pressures dropping rapidly. This happens when the ice is close enough to the rock for intermolecular disjoining pressures to become important, as demonstrated by the dot-dashed curve in Figure $4 \mathrm{~b}$. In the third stage, the system tends to an equilibrium where the liquid pressure vanishes (i.e. there is no water flow) and hence the solid pressure is simply the disjoining pressure. The radii of the ice and the cavity tend to the equilibrium values

$$
R_{\mathrm{eq}}=R_{0}+\frac{\rho_{\mathrm{s}} L \Delta T R_{0}}{E T_{\mathrm{m}}}
$$



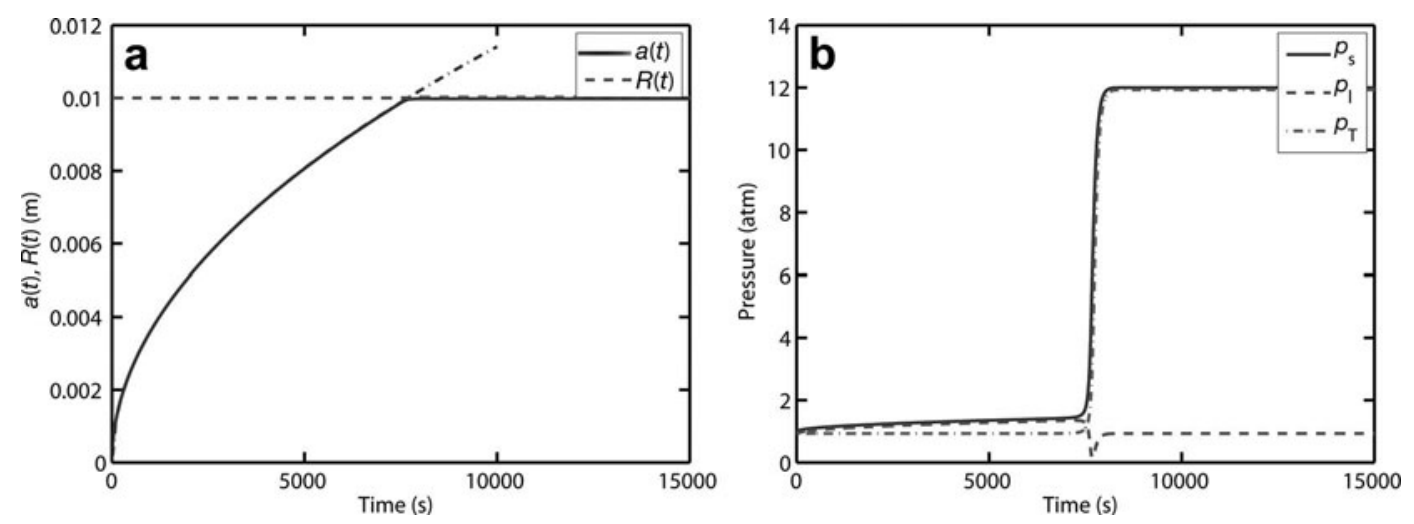

Fig. 5. Results for the evolution of (a) radii and (b) pressure values for a rock of intermediate permeability, such as sandstone. (a) The radius of the cavity is represented by the dashed curve, the radius of the ice by the solid curve, and the dot-dashed curve shows the approximate result from the expansion regime. (b) The solid curve is the solid pressure, the dashed curve is the water pressure and the dot-dashed curve the disjoining pressure.

and

$$
a_{\text {eq }}=R_{\text {eq }}-\left(\frac{A T_{\mathrm{m}}}{6 \pi \rho_{\mathrm{s}} L \Delta T}\right)^{1 / 3} .
$$

We next present the results for a rock of intermediate permeability $\sim 10^{-12} \mathrm{~cm}^{2}$ (e.g. a sandstone) (Fig. 5). We notice that in the first stage the cavity expansion is negligible, while the evolution of the solid ice agrees very well with the $t^{1 / 2}$ behaviour (dot-dashed curve) predicted above. There is no pressure difference across the solidification front since we ignore curvature effects and the disjoining forces are negligible (dot-dashed curve). Note that the maximum pressure during the expansion regime is much smaller than the maximum disjoining pressure. The second region is characterized by a very fast increase in the disjoining forces. The ice is now close enough to the cavity for intermolecular forces to become important, and the solidification process has slowed down considerably since the ice is limited by the cavity boundary. Disjoining forces cause the cavity to expand and water is sucked into the gap, as can be seen from the drop in the water pressure, which falls below the reference pressure $p_{\infty}=1 \mathrm{~atm}$. The third and last region is the recovery phase, where both the cavity and the ice keep growing until they reach (asymptotically) the stable state where the restoring force of the

elastic rock balances the disjoining pressure and there is no water flow.

The predicted water pressures could become negative, since the mathematical model does not allow the water to vaporize. An example of this can be seen in Figure 6, where we show the results for a limestone of typical permeability $\sim 10^{-13} \mathrm{~cm}^{2}$. Qualitatively, the results are similar to the results for sandstone (Fig. 5). The main difference is that the minimum water pressure is now negative. Of course, the pressures cannot actually have negative values. In fact, their values cannot be lower than the water vapour pressure. What is happening is that the lower permeability (compared to the sandstone case) does not allow water to flow towards the solidification front as quickly to fill the opening gap. As the pressure in, and close to, the pre-melted film becomes more and more negative, vapour bubbles can begin to form. The bubbles are, of course, unlikely to form inside the premelted film, which is only a few nanometres thick, smaller than the critical nucleation radius for a vapour bubble. However, bubbles can form in the rock pores close to the pre-melted film.

Above we mentioned that, although we have used an unrealistically large value of the Hamaker constant in our calculations, the main features of the results remain the same. More specifically, the difference is that a lower value
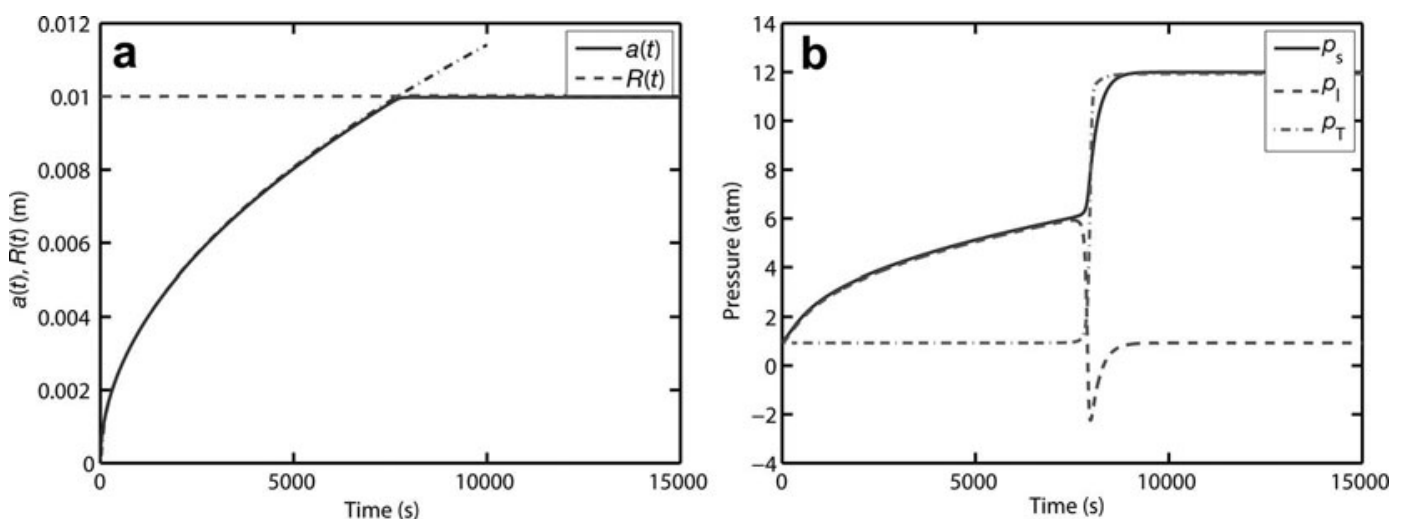

Fig. 6. Results for the evolution of (a) radii and (b) pressure values in a less permeable porous rock, such as limestone. (a) The radius of the cavity is represented by the dashed curve, the radius of the ice by the solid curve, and the dot-dashed curve shows the approximate result from the expansion regime. (b) The solid curve is the solid pressure, the dashed curve is the water pressure and the dot-dashed curve the disjoining pressure. The calculations give an unphysical negative water pressure. 
of the Hamaker constant reduces the time for which water pressures are below the reference pressure but also reduces the minimum value of the water pressure. Hence, the negative peak seen in Figure $6 b$ is deeper and sharper for lower values of $A$, and the mathematics predicts negative pressures for a larger spectrum of permeabilities. Similarly, our choice of $1 \mathrm{~cm}$ for the initial radius of the cavity is not essential for our conclusions to hold. A smaller cavity radius would make the expansion effect even less important and slightly decrease the negative pressure peak and increase the relaxation time for the liquid pressure. Importantly, the qualitative features remain the same.

\section{CONCLUSION}

We have studied the process of water freezing inside a spherical cavity and how the growth of ice can create high pressures inside the cavity. We have identified the main features of the process: the expansion of the water when it freezes and the disjoining forces across a thin pre-melted film that develop when the ice has grown to be close enough to the rock. We have compared the effect that these two processes have on the pressure inside the cavity and seen how they depend on the permeability of the rock. It is only in the case of very impermeable rocks, like granite, that expansion can raise the pressure enough to cause a fracture. For more permeable rocks, the water can escape the cavity easily during solidification, without experiencing any influence of the porous medium. Therefore, the main rise in pressure comes from the pre-melting stage when the ice is very close to the rock. In this case, the flow reverses and the water flows towards the solidification front. This is a result of disjoining forces that push the ice and the rock apart and cause the water pressure to drop. These disjoining forces can easily reach several atmospheres, even for very small undercoolings, indicating that they could be responsible for the fracturing of rocks.

Although the work presented here is for a simple geometry, it provides insight into the processes that take place during the freezing of water-saturated rocks. It demonstrates the relative importance of expansion and pre-melting and shows that, in most cases, high pressures occur at a later stage where the ice has extended to the cavity boundary, thus justifying focusing future attention on an ice-filled cavity. The idealized model developed here provides us with some conclusions about the importance of the different interactions taking place inside the cavity. These conclusions can work as a basis for a mathematical analysis of more complicated and realistic geometries.

\section{REFERENCES}

Bear, J. 1988. Dynamics of fluids in porous media. Mineola, NY, Dover.

Löwen, H., T. Beier and H. Wagner. 1989. Van der Waals theory of surface melting. Europhys. Lett., 9(8), 791-796.

Murton, J.B., R. Peterson and J.C. Ozouf. 2006. Bedrock fracture by ice segregation in cold regions. Science, 314(5802), 1127-1129.

Rempel, A.W., J.S. Wettlaufer and M.G. Worster. 2004. Premelting dynamics in a continuum model of frost heave. J. Fluid Mech., 498, 227-244.

Taber, S. 1929. Frost heaving. J. Geol., 37(5), 428-461.

Taber, S. 1930. The mechanics of frost heaving. J. Geol., 38(4), 303-317.

Walder, J. and B. Hallet. 1985. A theoretical model of the fracture of rock during freezing. Geol. Soc. Am. Bull., 96(3), 336-346.

Washburn, A.L. 1980. Geocryology: a survey of periglacial processes and environments. New York, etc., John Wiley and Sons.

Wettlaufer, J.S. and M.G. Worster. 2006. Premelting dynamics. Annu. Rev. Fluid Mech., 38, 427-452. 